\title{
Baustelle «nationales Qualitätsinstitut»
}

Wie kann die Ärzteschaft das künftige Schweizerische Institut für Qualität in der Gesundheitsversorgung mitgestalten? Dies war eine der wichtigen Fragen, die Nationalrätin Bea Heim und BAG-Vizedirektor Andreas Faller am letzten Treffen der Qualitätsverantwortlichen der in der Ärztekammer vertretenen Organisationen diskutierten. Seit 2008 sorgt diese von der FMH geschaffene Plattform dafür, dass die Ärzteschaft mitredet, wenn es um Qualität in der Medizin geht.

Fabienne Hohl

Korrespondenz: FMH

Martina Hersperger Daten, Demographie und Qualität DDQ Elfenstrasse 18 Postfach 170 CH-3000 Bern 15 ddq@fmh.ch
Aus allen Ecken der Schweiz sind am 9. Juni 2011 rund 30 Qualitätsverantwortliche von ärztlichen Fach- und Dachorganisationen wie auch von kantonalen Ärztegesellschaften nach Bern gereist, um sich über die politischen und fachlichen Entwicklungen im Bereich der medizinischen Qualität zu informieren und auszutauschen. Sie tun dies auf Einladung der FMH seit über drei Jahren im Bewusstsein, dass Ärztinnen und Ärzte in Qualitätsfragen eine aktive und sichtbare Rolle spielen müssen. «Wir wollen wissen, was vonseiten des BAG und Bundes läuft», sagt Cornelia Moll-Roth von der Gesellschaft der Ärztinnen und Ärzte des Kantons Solothurn. «Denn sicher ist: Wir Leistungsbringer wollen und müssen die medizinische Qualität mitdefinieren. Es wird in Sachen Qualitätssicherung schon sehr vieles geleistet, aber es muss noch mehr - auch die von der Öffentlichkeit geforderte - Transparenz in der Gesundheitsversorgung geschaffen werden.»

Hochaktuell ist in diesem Zusammenhang die geplante Gründung eines Schweizerischen Instituts für Qualität in der Gesundheitsversorgung im Rahmen der nationalen Qualitätsstrategie des Bundes. Am letzten Treffen der Qualitätsverantwortlichen skizzierten SP-Nationalrätin Bea Heim, BAG-Vizedirektor Andreas Faller und FMH-Zentralvorstand Daniel Herren Ziele und Realitäten dieses wichtigen nationalen Projekts.

\section{Nationalrätin Heim: «Qualitätsinstitut mit Patientennutzen»}

Einig waren sich alle drei: Es braucht ein nationales Qualitätsinstitut, um den wachsenden ökonomischen Druck auf das Gesundheitswesen wissenschaftlich fundiert zu beobachten und ihm nötigenfalls entgegenzuwirken. Das geplante Institut soll die Grundlagen für evidenzbasierte Entscheide schaffen, denn Qualitätssicherung verbessert medizinische Behandlungen und Patientensicherheit. Nicht zuletzt hilft sie, unnötige Kosten in der Gesundheitsversorgung zu vermeiden. Doch zurzeit mangle es noch an Wissen und politischem Konsens, was Qualität in der Gesundheitsversorgung alles umfasse, stellt Nationalrätin Bea Heim fest. Deshalb müsse der Bund gemeinsam mit der Ärzteschaft und den übrigen Gesundheitsakteuren nationale Qualitätsziele definieren und

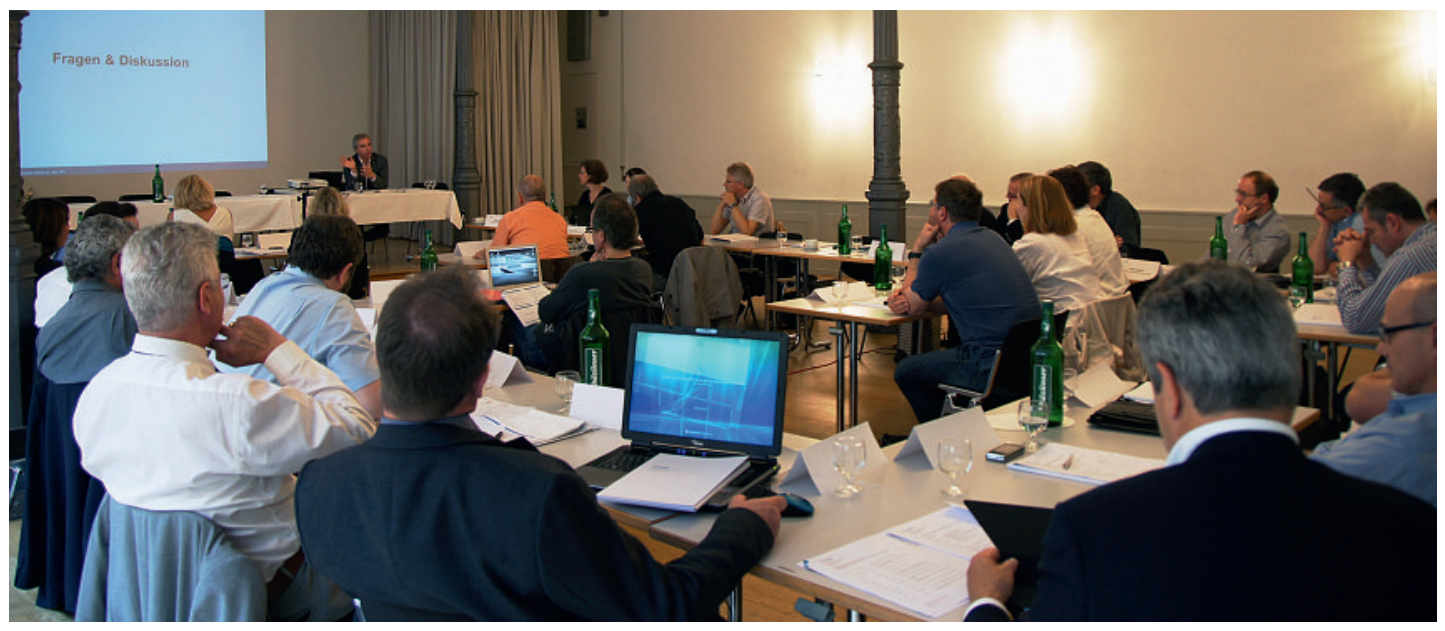

Die Qualitätsverantwortlichen der Ärztegesellschaften nutzen die Gelegenheit zur Diskussion. 
durchsetzen, fordert sie. «Dafür muss der Bund die politischen Voraussetzungen schaffen und die Ärzteschaft die medizinische Verantwortung tragen. Sie stehen gemeinsam in der Führungsverantwortung, und zwar im Interesse der Patienten. Keinesfalls dürfen hier die rein ökonomischen Kriterien der Kassen zum Zuge kommen!» Heim regt einen paritätischen Institutsrat an, in dessen Auftrag der Bund agieren soll. Denn für sie ist klar: «Was zählt, ist die Fachlichkeit.» Um diese zu gewährleisten, sei die Politik aber auf die Unterstützung der Ärzteschaft angewiesen, appelliert die Nationalrätin an die Qualitätsverantwortlichen. «Es ist leider viel einfacher, vom Parlament Geld für Kampfjets zu bekommen als für die Sicherheit der Patienten und die Sicherung der Behandlungs- und Versorgungsqualität in der Medizin.» Heims Vision des nationalen Qualitätsinstituts orientiert sich am maximalen Patientennutzen, weshalb sie das Fehlen von patientenzentrierter Forschung in der Auftragsbeschreibung des Instituts moniert.

\section{Ball beim BAG}

Zugegeben, angesichts der Partikularinteressen und der Komplexität des Themas Qualität steht das Bundesamt für Gesundheit (BAG) mit der Schaffung eines nationalen Qualitätsinstituts vor einer grossen Aufgabe. Und sie wird nicht einfacher, weil «die gesetzlichen Grundlagen schon seit längerer Zeit im KVG vorhanden sind», wie BAG-Vizedirektor Andreas Faller bekennt. Doch plädiert er energisch für Zukunftsorientierung in der Zusammenarbeit der Gesundheitsakteure, damit die nationale Qualitätsstrategie unter dem Lead des Bundes in die benötigte Transparenz für alle Zielgruppen - die Bevölkerung eingeschlossen - mündet. Der Weg dazu führt laut Faller über das nationale Qualitätsinstitut, die gemeinsame Rollenklärung aller Gesundheitspartner, Monitoring, Controlling sowie die Umsetzung von Qualitätsprogrammen. Für die Gestaltung des Schweizerischen Instituts für Qualität liegen zwei Vorschläge auf dem Tisch [1]. Derzeit prüft das BAG, ob es eine Änderung des KVG braucht, um das Institut in der angedachten Form realisieren zu können.

\section{FMH will unabhängiges Qualitätsinstitut}

FMH-Zentralvorstand Daniel Herren begrüsst die Zielsetzung des Instituts als Dachorganisation, Rahmenbedingungen für die Qualitätssicherung in der Medizin festzulegen, fordert aber klar dessen Unabhängigkeit: «Das Qualitätsinstitut darf keine weitere Abteilung des BAG sein», so Herren. Und er warnt eindringlich vor einer weiteren Redimensionierung der heute jährlich veranschlagten 25 Millionen für die gesamte nationale Qualitätsstrategie. «Mit 10 Millionen ist nichts Sinnvolles realisierbar.» Herren hakt auch beim Thema Health Assessment Technology (HTA) nach, insbesondere bezüglich der oft langwierigen Zulassungsverfahren für neue Medikamente. Bis dato sei unklar, wie dieses Prozedere schlanker wer-

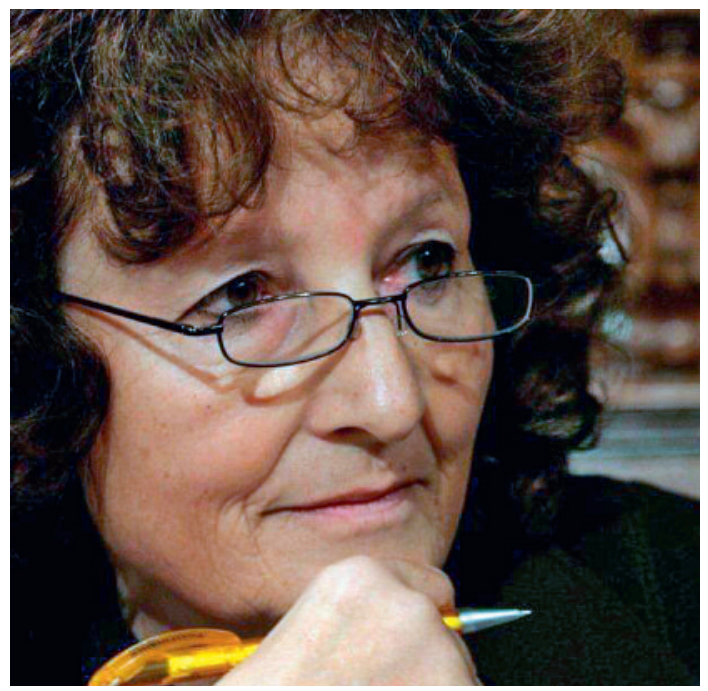

Für das geplante nationale Qualitätsinstitut setzt SPNationalrätin Bea Heim auf die Mitwirkung der Ärzteschaft.

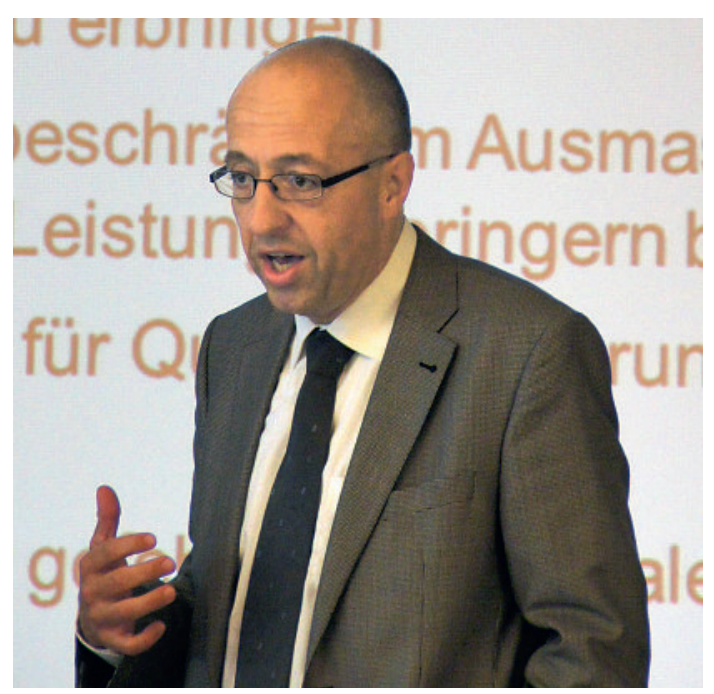

BAG-Vizedirektor Andreas Faller wirbt für eine zukunftsorientierte Zusammenarbeit der Gesundheitsakteure im Qualitätsbereich.

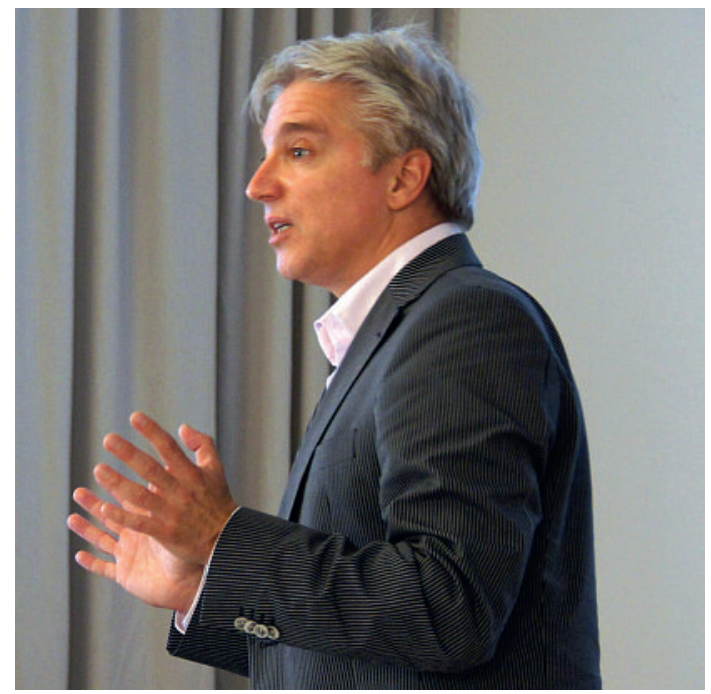

Fordert ein unabhängiges künftiges nationales Qualitätsinstitut: FMH-Zentralvorstand Daniel Herren. 
Treffen der Qualitätsverantwortlichen

Seit 2008 versammelt die Verbindung der Schweizer Ärztinnen und Ärzte FMH die Qualitätsverantwortlichen der Ärztegesellschaften zweimal jährlich an den «Q-Treffen». Ziel der Plattform ist es, die Qualitätsverantwortlichen bei der kompetenten Gestaltung medizinischer Qualitätsarbeit zu unterstützen, in fachlicher und politischer Hinsicht sowie in Zusammenarbeit mit den Gesundheitspartnern. An den Q-Treffen informiert die Abteilung Daten, Demographie und Qualität (DDQ) der FMH über laufende Qualitätsprojekte und fördert den Austausch zu aktuellen Qualitätsfragen. Stets präsentieren auch namhafte Referierende aus Wissenschaft und Gesundheitspolitik aktuelles Wissen über Qualitätssicherung. Die Q-Treffen ermöglichen, Fragen, Bedürfnisse und Anregungen zur Qualität in der Medizin innerhalb und ausserhalb der Ärzteschaft zu artikulieren, wodurch alle Beteiligten wichtige Impulse erhalten.

Die Q-Treffen sind eine Veranstaltungsreihe der Arbeitsgruppe Qualität der FMH (AGQ-FMH) und werden organisatorisch von der Abteilung DDQ der FMH (verantwortlicher Zentralvorstand: Dr. med. Daniel Herren, Leiterin: Martina Hersperger) betreut. Das nächste Treffen der Qualitätsverantwortlichen findet am 10 . November 2011 statt.

Weitere Informationen zu den Qualitätsprojekten der FMH: www.fmh.ch $\rightarrow$ Qualität.

den könne. Faller ist bewusst, dass die BAG-Prüfung von Innovationen «verbesserungsfähig» sei, und einige dem Gesetz zugrundeliegenden ethischen Überlegungen teilweise zu strikt, wie beispielsweise im Bereich der Zulassung von pädiatrischen Arzneimitteln gemäss Heilmittelgesetz, auf die Kinder- und Jugendpsychiaterin Hélène Beutler hinweist. Hans Ulrich Iselin von der Kantonalen Ärztegesellschaft Aargau fordert, HTA möglichst ins geplante Qualitätsinstitut zu integrieren, um Synergien zu nutzen. Überhaupt entspinnt sich eine angeregte Plenumsdiskussion, bei der sich Faller als aufmerksamer Gesprächspartner herausstellt, der willens scheint, die Anliegen der Ärzteschaft ins BAG zu tragen. So auch bei der Frage nach medizinischen Registern, die Onkologe Jürg Nadig anspricht. Faller versichert, dass der Bund bei der Erstellung von Registern in Mitwirkungspflicht stehe - gerade hinsichtlich der Finanzierung durch die In- dustrie, die dafür im Gegenzug Einsicht in die Register fordere [2]. Ein Thema, das unbedingt im künftigen Registergesetz geregelt gehöre, wie Daniel Herren präzisiert.

\section{Aktive Ärzteschaft}

Bei aller Offenheit, die BAG-Vizedirektor Faller signalisiert - die versammelten Qualitätsverantwortlichen der Ärztegesellschaften wünschen sich neben Worten vor allem auch Taten. «Wir wissen nicht, wie wir konkret an der Umsetzung der nationalen Qualitätsstrategie mitarbeiten können», konstatiert Psychiaterin Yvette Attinger Andreoli. Es scheint, dass bei der Qualität in der Medizin, wie überall in der schweizerischen Demokratie, nur das Prinzip des langen Atems Erfolg verspricht. Die FMH trägt Fundiertes zur Diskussion bei und gestaltet sie zielgerichtet im Sinne der Ärzteschaft: indem sie Studien anregt, mitträgt und darüber informiert, wie beispielsweise im Falle der Evaluation des Informationsbedarfs von Patienten zur Qualität in der hausärztlichen Leistungserbringung (2010) [3]; indem sie den Swiss Quality Award mitgestaltet und sich für Qualitätsinnovationen im Gesundheitswesen einsetzt [4]; oder indem sie mit Q-Monitoring ein niederschwelliges Projekt lanciert, das die bereits heute durchgeführten Qualitätsaktivitäten der ambulant tätigen Ärzteschaft sichtbar macht [5]. Die engagierten Voten am Qualitätstreffen der FMH zeigen: Die Ärzteschaft hat viel zur nationalen Qualitätsstrategie beizutragen, und das Interesse der Ärzteorganisationen gibt der FMH den nötigen Rückhalt, das Fachwissen ihrer Mitglieder auf nationaler Ebene einzubringen.

\section{Literatur}

1 Bericht an den Bundesrat zur Konkretisierung der Qualitätsstrategie des Bundes im Schweizerischen Gesundheitswesen», Version 25. Mai 2011; www.bag. admin.ch $\rightarrow$ Themen $\rightarrow$ Krankenversicherung $\rightarrow$ Aktuell $\rightarrow$ Archiv «Aktuell» $\rightarrow$ 25.5.2011.

2 Unternehmen können per Gesetz zur Finanzierung von Registern verpflichtet werden, wenn medizinische Leistungen neu in die Grundversicherung aufgenommen werden sollen.

3 Schläpfer L. Hausärzte, wie Patienten sie sich wünschen. Schweiz Ärztezeitung. 2010;91(26/27):1022-3.

4 Beutler J, Scholer M. Swiss Quality Award: die Gewinner 2011. Schweiz Ärztezeitung. 2011;92(22):833-5. www.swissqualityaward.ch

5 Nikolic V. Die Ärzteschaft sichert Qualität - und wie! Schweiz Ärztezeitung. 2010;92(25):973-6. 